International Journal of Information Technology Convergence and Services (IJITCS) Vol.2, No.3, June 2012

\title{
SLIDING MODE CONTROLLER DESIGN FOR THE ANTI-SYNCHRONIZATION OF IDENTICAL HYPERCHAOTIC NEWTON-LEIPNIK SYSTEMS
}

\author{
Sundarapandian Vaidyanathan ${ }^{1}$ \\ ${ }^{1}$ Research and Development Centre, Vel Tech Dr. RR \& Dr. SR Technical University \\ Avadi, Chennai-600 062, Tamil Nadu, INDIA \\ sundarvtulgmail.com
}

\begin{abstract}
This paper investigates the anti-synchronization of identical hyperchaotic Newton-Leipnik systems (Ghosh and Bhattacharya, 2010) via sliding mode control. The stability results derived in this paper for the anti-synchronization of identical hyperchaotic Xu systems are established using Lyapunov stability theory. Numerical simulations are shown to illustrate and validate the anti-synchronization schemes derived in this paper for the identical hyperchaotic Newton-Leipnik systems.
\end{abstract}

\section{KEYWORDS}

Sliding Mode Control, Anti-Synchronization, Hyperchaotic Systems, Hyperchaotic Newton-Leipnik System.

\section{INTRODUCTION}

Hyperchaotic system is usually defined as a chaotic system with more than one positive Lyapunov exponent. The first hyperchaotic system was discovered by O.E. Rössler ([1], 1979). Since hyperchaotic system has the characteristics of high capacity, high security and high efficiency, it has the potential of broad applications in nonlinear circuits, secure communications, lasers, neural networks, biological systems and so on. Thus, the studies on hyperchaotic systems, viz. control, synchronization and circuit implementation are very challenging problems in the chaos literature.

In the problem of chaos synchronization, the master-slave formalism is commonly adopted. If a certain chaotic system is called the master or drive system and another system is called the slave or response system, then the goal of the anti-synchronization is to apply the output of the master system so as to control the slave system so that the states of the slave system have the same amplitude but opposite signs as the states of the master system asymptotically.

Since the seminal paper published by Pecora and Carroll ([2], 1990), chaos synchronization problem has been studied rigorously in the chaos literature [2-17]. Chaos theory has been applied to a variety of applied fields such as physical systems [3], chemical systems [4], ecological systems [5], secure communications [6-8], etc.

In the last two decades, various synchronization schemes have been successfully deployed for global synchronization such as PC method [2], OGY method [9], active control method [10-14], adaptive control method [15-20], time-delay feedback method [21], backstepping design method [22], sampled-data feedback method [23], etc. 
International Journal of Information Technology Convergence and Services (IJITCS) Vol.2, No.3, June 2012

In this paper, we derive new results based on the sliding mode control [24-28] for the antisynchronization of identical hyperchaotic Newton-Leipnik systems ([29], Ghosh and Bhattacharya, 2010).

This paper has been organized as follows. In Section 2, we present the problem statement of anti-synchronization and detail our methodology using sliding mode control (SMC). In Section 3 , we discuss the anti- synchronization of identical hyperchaotic Newton-Leipnik systems. In Section 4, we conclude with a summary of the main results derived in this paper using SMC.

\section{Problem Statement and Our Sliding Mode Control Design}

Consider the chaotic system described by

$$
\dot{x}=A x+f(x)
$$

where $x \in R^{n}$ is the state of the system, $A$ is the $n \times n$ matrix of the system parameters and $f: R^{n} \rightarrow R^{n}$ is the nonlinear part of the system.

We consider the system (1) as the master or drive system.

As the slave or response system, we consider the following chaotic system described by the dynamics

$$
\dot{y}=A y+f(y)+u
$$

where $y \in R^{n}$ is the state of the system and $u \in R^{m}$ is the controller to be designed.

If we define the anti-synchronization error as

$$
e=y+x,
$$

then the error dynamics is obtained as

$$
\dot{e}=A e+\eta(x, y)+u,
$$

where

$$
\eta(x, y)=f(y)+f(x)
$$

The objective of the anti-synchronization problem is to find a controller $u$ such that

$$
\lim _{t \rightarrow \infty}\|e(t)\|=0 \text { for all } e(0) \in R^{n} .
$$

To solve this problem, we first define the control $u$ as

$$
u=-\eta(x, y)+B v
$$

where $B$ is a constant gain vector selected such that $(A, B)$ is controllable. 
Substituting (6) into (4), the error dynamics simplifies to

$$
\dot{e}=A e+B v
$$

which is a linear time-invariant control system with single input $v$.

Thus, the original anti-synchronization problem can be replaced by an equivalent problem of stabilizing the zero solution $e=0$ of the system (7) by a suitable choice of the sliding mode control. In the sliding mode control, we define the variable

$$
s(e)=C e=c_{1} e_{1}+c_{2} e_{2}+\cdots+c_{n} e_{n}
$$

where

$$
C=\left[\begin{array}{llll}
c_{1} & c_{2} & \cdots & c_{n}
\end{array}\right]
$$

is a constant vector to be determined.

In the sliding mode control, we constrain the motion of the system (7) to the sliding manifold defined by

$$
S=\left\{x \in R^{n} \mid s(e)=0\right\}
$$

which is required to be invariant under the flow of the error dynamics (7).

When in sliding manifold $S$, the system (7) satisfies the following conditions:

$$
s(e)=0
$$

which is the defining equation for the manifold $S$ and

$$
\dot{s}(e)=0
$$

which is the necessary condition for the state trajectory $e(t)$ of (7) to stay on the sliding manifold $S$.

Using (7) and (8), the equation (10) can be rewritten as

$$
\dot{s}(e)=C[A e+B v]=0
$$

Solving (11) for $v$, we obtain the equivalent control law

$$
v_{\mathrm{eq}}(t)=-(C B)^{-1} C A e(t)
$$

where $C$ is chosen such that

$$
C B \neq 0 \text {. }
$$


Substituting (12) into the error dynamics (7), we obtain the closed-loop dynamics as

$$
\dot{e}=\left[I-B(C B)^{-1} C\right] A e
$$

The row vector $C$ is selected such that the system matrix of the controlled dynamics $\left[I-B(C B)^{-1} C\right] A$ is Hurwitz, i.e. it has all eigenvalues with negative real parts. Then the controlled system (13) is globally asymptotically stable.

To design the sliding mode controller for (7), we apply the constant plus proportional rate reaching law

$$
\dot{s}=-q \operatorname{sgn}(s)-k s
$$

where $\operatorname{sgn}(\cdot)$ denotes the sign function and the gains $q>0, k>0$ are determined such that the sliding condition is satisfied and sliding motion will occur.

From equations (11) and (14), we can obtain the control $v(t)$ as

$$
v(t)=-(C B)^{-1}[C(k I+A) e+q \operatorname{sgn}(s)]
$$

which yields

$$
v(t)= \begin{cases}-(C B)^{-1}[C(k I+A) e+q], & \text { if } s(e)>0 \\ -(C B)^{-1}[C(k I+A) e-q], & \text { if } s(e)<0\end{cases}
$$

Theorem 2.1. The master system (1) and the slave system (2) are globally and asymptotically anti-synchronized for all initial conditions $x(0), y(0) \in R^{n}$ by the feedback control law

$$
u(t)=-\eta(x, y)+B v(t)
$$

where $v(t)$ is defined by $(15)$ and $B$ is a column vector such that $(A, B)$ is controllable. Also, the sliding mode gains $k$, $q$ are positive.

Proof. First, we note that substituting (17) and (15) into the error dynamics (4), we obtain the closed-loop error dynamics as

$$
\dot{e}=A e-B(C B)^{-1}[C(k I+A) e+q \operatorname{sgn}(s)]
$$

To prove that the error dynamics (18) is globally asymptotically stable, we consider the candidate Lyapunov function defined by the equation

$$
V(e)=\frac{1}{2} s^{2}(e)
$$

which is a positive definite function on $R^{n}$. 
Differentiating $V$ along the trajectories of (18) or the equivalent dynamics (14), we get

$$
\dot{V}(e)=s(e) \dot{s}(e)=-k s^{2}-q \operatorname{sgn}(s) s
$$

which is a negative definite function on $R^{n}$.

Thus, by Lyapunov stability theory [30], we conclude the error dynamics (18) is globally asymptotically stable for all initial conditions $e(0) \in R^{n}$.

Hence, it is immediate that the master system (1) and the slave system (2) are globally and asymptotically anti-synchronized for all initial conditions $x(0), y(0) \in R^{n}$.

This completes the proof.

\section{Anti-Synchronization of Identical Hyperchaotic NeWTON- LEIPNIK SySTEMS VIA SLIDING MODE CONTROL}

\subsection{Theoretical Results}

Here, the master system is described by the hyperchaotic Newton-Leipnik dynamics (2010)

$$
\begin{aligned}
& \dot{x}_{1}=-a x_{1}+x_{2}+10 x_{2} x_{3}+x_{4} \\
& \dot{x}_{2}=-x_{1}-0.4 x_{2}+5 x_{1} x_{3} \\
& \dot{x}_{3}=b x_{3}-5 x_{1} x_{2} \\
& \dot{x}_{4}=-c x_{1} x_{3}+d x_{4}
\end{aligned}
$$

where $x_{1}, x_{2}, x_{3}, x_{4}$ are state variables and $a, b, c, d$ are positive, constant parameters of the system.

The 4D system (21) is hyperchaotic when the parameters are chosen as

$$
a=0.4, b=0.175, c=0.8 \text { and } d=0.01 \text {. }
$$

Figure 1 illustrates the phase portrait of the hyperchaotic Newton-Leipnik system.

The slave system is described by the controlled hyperchaotic Newton-Leipnik dynamics

$$
\begin{aligned}
& \dot{y}_{1}=-a y_{1}+y_{2}+10 y_{2} y_{3}+y_{4}+u_{1} \\
& \dot{y}_{2}=-y_{1}-0.4 y_{2}+5 y_{1} y_{3}+u_{2} \\
& \dot{y}_{3}=b y_{3}-5 y_{1} y_{2}+u_{3} \\
& \dot{y}_{4}=-c y_{1} y_{3}+d y_{4}+u_{4}
\end{aligned}
$$

where $y_{1}, y_{2}, y_{3}, y_{4}$ are state variables and $u_{1}, u_{2}, u_{3}, u_{4}$ are the controllers to be designed. 

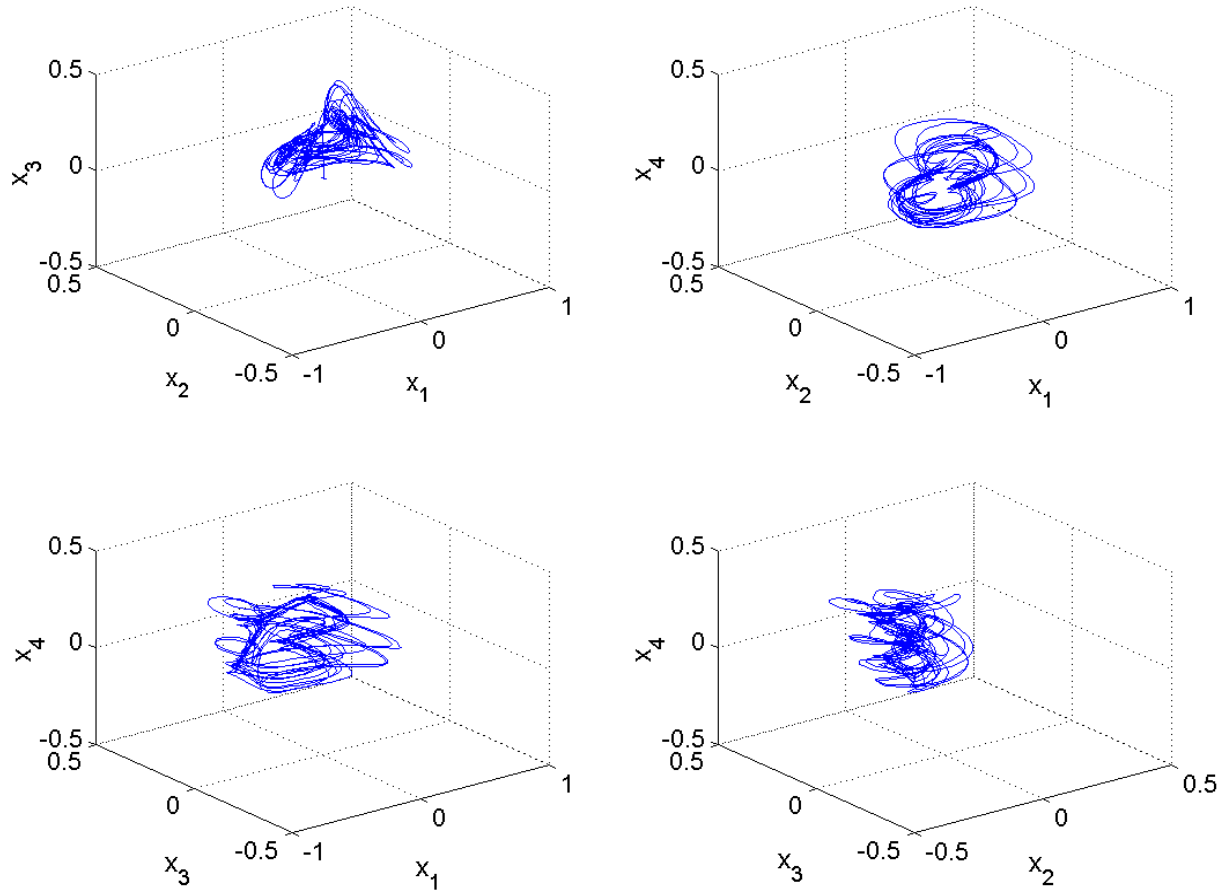

Figure 1. Phase Portrait of the Hyperchaotic Newton-Leipnik System

The chaos anti-synchronization error is defined by

$$
e_{i}=y_{i}+x_{i}, \quad(i=1,2,3,4)
$$

The error dynamics is easily obtained as

$$
\begin{aligned}
& \dot{e}_{1}=-a e_{1}+e_{2}+e_{4}+10\left(y_{2} y_{3}+x_{2} x_{3}\right)+u_{1} \\
& \dot{e}_{2}=-e_{1}-0.4 e_{2}+5\left(y_{1} y_{3}+x_{1} x_{3}\right)+u_{2} \\
& \dot{e}_{3}=b e_{3}-5\left(y_{1} y_{2}+x_{1} x_{2}\right)+u_{3} \\
& \dot{e}_{4}=d e_{4}-c\left(y_{1} y_{3}+x_{1} x_{3}\right)+u_{4}
\end{aligned}
$$

We write the error dynamics (24) in the matrix notation as

$$
\dot{e}=A e+\eta(x, y)+u
$$

where

$$
A=\left[\begin{array}{cccc}
-a & 1 & 0 & 1 \\
-1 & -0.4 & 0 & 0 \\
0 & 0 & b & 0 \\
0 & 0 & 0 & d
\end{array}\right], \quad \eta(x, y)=\left[\begin{array}{c}
10\left(y_{2} y_{3}+x_{2} x_{3}\right) \\
5\left(y_{1} y_{3}+x_{1} x_{3}\right) \\
-5\left(y_{1} y_{2}+x_{1} x_{2}\right) \\
-c\left(y_{1} y_{3}+x_{1} x_{3}\right)
\end{array}\right]
$$


and

$$
u=\left[\begin{array}{l}
u_{1} \\
u_{2} \\
u_{3} \\
u_{4}
\end{array}\right] .
$$

First, we set $u$ as

$$
u=-\eta(x, y)+B v
$$

where $B$ is taken such that $(A, B)$ is controllable.

We choose $B$ as

$$
B=\left[\begin{array}{l}
1 \\
1 \\
1 \\
1
\end{array}\right]
$$

In the hyperchaotic case, the parameter values are taken as

$$
a=0.4, b=0.175, c=0.8 \text { and } d=0.01 \text {. }
$$

The sliding mode variable is selected as

$$
s=C e=\left[\begin{array}{llll}
2 & 2 & 3 & 0
\end{array}\right] e=2 e_{1}+2 e_{2}+3 e_{3}
$$

which makes the sliding mode state equation asymptotically stable.

We choose the sliding mode gains as

$$
k=6 \text { and } q=0.2 \text {. }
$$

We note that a large value of $k$ can cause chattering and an appropriate value of $q$ is chosen to speed up the time taken to reach the sliding manifold as well as to reduce the system chattering.

From Eq. (15), we can obtain $v(t)$ as

$$
v(t)=-1.3143 e_{1}-1.8857 e_{2}-2.6464 e_{3}-0.2857 e_{4}-0.0286 \operatorname{sgn}(s)
$$

Thus, the required sliding mode controller is obtained as

$$
u=-\eta(x, y)+B v
$$

where $\eta(x, y), B$ and $v(t)$ are given by the equations (26), (28) and (31). 
By Theorem 2.1, we obtain the following result.

Theorem 3.1. The identical hyperchaotic Newton-Leipnik systems (21) and (22) are globally and asymptotically anti-synchronized for all initial conditions with the sliding mode controller $u$ defined by (32).

\subsection{Numerical Results}

For the numerical simulations, the fourth-order Runge-Kutta method with time-step $h=10^{-8}$ is adopted to solve the hyperchaotic Newton-Leipnik systems (21) and (22) with the sliding controller $u$ given by (32) using MATLAB.

In the hyperchaotic case, the parameter values are given by $a=0.4, b=0.175, c=0.8$ and $d=0.01$. The sliding mode gains are chosen as $k=6$ and $q=0.2$.

The initial values of the master system (21) are taken as

$$
x_{1}(0)=9, x_{2}(0)=6, x_{3}(0)=-7, \quad x_{4}(0)=-12
$$

The initial values of the slave system (22) are taken as

$$
y_{1}(0)=5, y_{2}(0)=-18, y_{3}(0)=20, y_{4}(0)=4
$$

Figure 2 depicts the time-history of the anti-synchronization errors $e_{1}, e_{2}, e_{3}, e_{4}$.

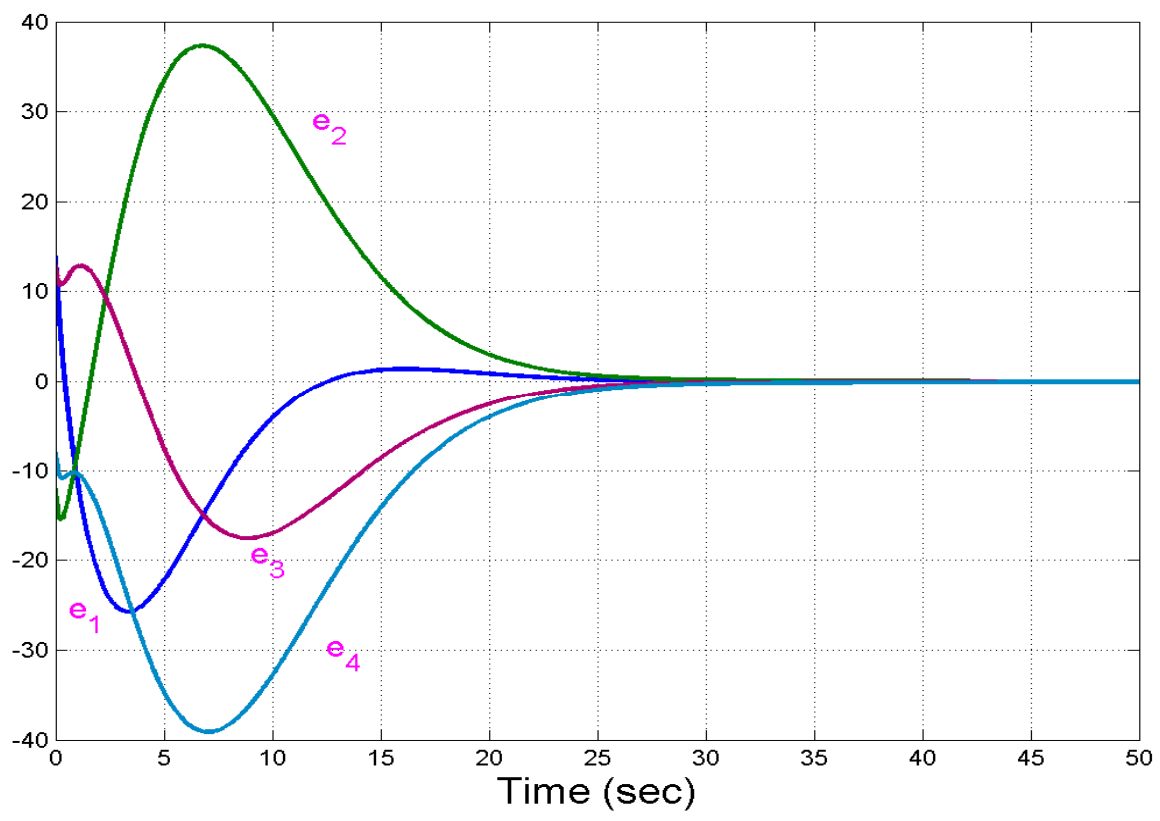

Figure 2. Time-History of the Anti-Synchronization Error 


\section{Conclusions}

In this paper, we have deployed sliding mode control (SMC) to achieve anti-synchronization for the identical hyperchaotic Newton-Leipnik systems (2010). Our anti-synchronization results for the identical hyperchaotic Newton-Leipnik systems have been proved using Lyapunov stability theory. Numerical simulations have been provided to show the effectiveness of the SMC-based anti-synchronization results derived for the hyperchaotic Newton-Leipnik systems.

\section{REFERENCES}

[1] Rössler, O.E. (1979) “An equation for hyperchaos”, Physics Letters, Vol. 71A, pp 155-157.

[2] Pecora, L.M. \& Carroll, T.L. (1990) "Synchronization in chaotic systems", Phys. Rev. Lett., Vol. 64, pp 821-824.

[3] Lakshmanan, M. \& Murali, K. (1996) Nonlinear Oscillators: Controlling and Synchronization, World Scientific, Singapore.

[4] Han, S.K., Kerrer, C. \& Kuramoto, Y. (1995) "Dephasing and burstling in coupled neural oscillators", Phys. Rev. Lett., Vol. 75, pp 3190-3193.

[5] Blasius, B., Huppert, A. \& Stone, L. (1999) "Complex dynamics and phase synchronization in spatially extended ecological system”, Nature, Vol. 399, pp 354-359.

[6] Cuomo, K.M. \& Oppenheim, A.V. (1993) "Circuit implementation of synchronized chaos with applications to communications,” Physical Review Letters, Vol. 71, pp 65-68.

[7] Kocarev, L. \& Parlitz, U. (1995) "General approach for chaotic synchronization with applications to communication,” Physical Review Letters, Vol. 74, pp 5028-5030.

[8] Tao, Y. (1999) "Chaotic secure communication systems - history and new results," Telecommun. Review, Vol. 9, pp 597-634.

[9] Ott, E., Grebogi, C. \& Yorke, J.A. (1990) "Controlling chaos", Phys. Rev. Lett., Vol. 64, pp 1196-1199.

[10] Ho, M.C. \& Hung, Y.C. (2002) "Synchronization of two different chaotic systems using generalized active network," Physics Letters A, Vol. 301, pp 424-428.

[11] Huang, L., Feng, R. \& Wang, M. (2005) "Synchronization of chaotic systems via nonlinear control," Physical Letters A, Vol. 320, pp 271-275.

[12] Chen, H.K. (2005) "Global chaos synchronization of new chaotic systems via nonlinear control," Chaos, Solitons \& Fractals, Vol. 23, pp 1245-1251.

[13] Sundarapandian, V. (2011) "Global chaos synchronization of four-scroll and four-wing chaotic attractors by active nonlinear control," International Journal on Computer Science and Engineering, Vol. 3, No. 5, pp. 2145-2155.

[14] Sundarapandian, V. (2011) "Global chaos synchronization of Li and Liu-Chen-Liu chaotic systems by active nonlinear control," International Journal of Advances in Science and Technology, Vol. 3, No. 1, pp. 1-12.

[15] Lu, J., Wu, X., Han, X. \& Lü, J. (2004) “Adaptive feedback synchronization of a unified chaotic system," Physics Letters A, Vol. 329, pp 327-333.

[16] Chen, S.H. \& Lü, J. (2002) "Synchronization of an uncertain unified system via adaptive control," Chaos, Solitons \& Fractals, Vol. 14, pp 643-647.

[17] Sundarapandian, V. (2011) "Adaptive control and synchronization of hyperchaotic Cai system", International Journal of Control Theory and Computer Modelling, Vol. 1, No. 1, pp 1-13.

[18] Sundarapandian, V. (2011) "Adaptive synchronization of hyperchaotic Lorenz and hyperchaotic Liu systems", International Journal of Instrumentation and Control Systems, Vol. 1, No. 1, pp $1-18$. 
[19] Sundarapandian, V. (2011) "Adaptive control and synchronization of Liu's four-wing chaotic system with cubic nonlinearity," International Journal of Computer Science, Engineering and Applications, Vol. 1, No. 4, pp 127-138.

[20] Sundarapandian, V. \& Karthikeyan, R. (2011) "Global chaos synchronization of Pan and Lü chaotic systems via adaptive control," International Journal of Information Technology, Convergence and Services, Vol. 1, No. 5, pp. 49-66.

[21] Park, J.H. \& Kwon, O.M. (2003) “A novel criterion for delayed feedback control of time-delay chaotic systems," Chaos, Solitons \& Fractals, Vol. 17, pp 709-716.

[22] Wu, X. \& Lü, J. (2003) "Parameter identification and backstepping control of uncertain Lü system," Chaos, Solitons \& Fractals, Vol. 18, pp 721-729.

[23] Zhao, J. \& J. Lu (2006) "Using sampled-data feedback control and linear feedback synchronization in a new hyperchaotic system," Chaos, Solitons \& Fractals, Vol. 35, pp 376382.

[24] Slotine, J.E. \& Sastry, S.S. (1983) "Tracking control of nonlinear systems using sliding surface with application to robotic manipulators," International Journal of Control, Vol. 38, pp 465-492.

[25] Utkin, V.I. (1993) "Sliding mode control design principles and applications to electric drives," IEEE Trans. Industrial Electronics, Vol. 40, pp 23-36, 1993.

[26] Sundarapandian, V. (2011) "Global chaos synchronization of four-wing chaotic systems by sliding mode control”, International Journal of Control Theory and Computer Modelling, Vol. 1, No. 1, pp 15-31.

[27] Sundarapandian, V. (2011) "Global chaos synchronization of Lü-Chen-Cheng four-scroll chaotic attractors by sliding mode control, Computer Science \& Engineering: An International Journal, Vol. 1, No. 3, pp 26-35.

[28] Sundarapandian, V. \& Sivaperumal, S. (2011) "Sliding mode controller design for global chaos synchronization of hyperchaotic Lorenz systems," Computer Science \& Engineering: An International Journal, Vol. 1, No.. 4, pp. 61-71.

[29] Ghosh, D. \& Bhattacharya, S. (2010) "Projective synchronization of new hyperchaotic system with fully unknown parameters", Nonlinear Dynamics, Vol. 61, pp 11-21.

[30] Hahn, W. (1967) The Stability of Motion, Springer, New York.

\section{Author}

Dr. V. Sundarapandian earned his Doctor of Science degree in Electrical and Systems Engineering from Washington University, St. Louis, USA in May 1996. He is a Professor at the R \& D Centre at Vel Tech Dr. RR \& Dr. SR Technical University, Chennai, Tamil Nadu, India. He has published over 260 papers in refereed International Journals. He has published over 170 papers in National and International Conferences. He is the Editor-in-Chief of the AIRCC Journals International Journal of Instrumentation and Control Systems, International Journal of Control Systems and Computer Modelling, and International Journal of Information Technology, Control and Automation. His research interests are Linear and Nonlinear Control Systems, Chaos Theory and Control, Soft Computing, Optimal Control, Operations Research, Mathematical Modelling and Scientific Computing. He has delivered several Key Note Lectures on Control Systems, Chaos Theory, Scientific Computing, Mathematical Modelling, MATLAB and SCILAB.

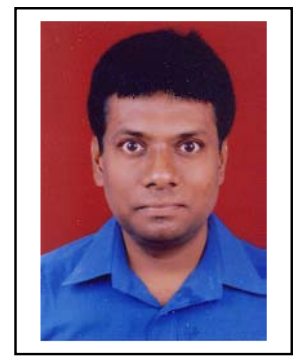

